\title{
Postoperative controlling nutritional status score is an independent risk factor of survival for patients with small hepatocellular carcinoma: a retrospective study
}

Wei Peng ${ }^{1,2}$, Minghong Yao ${ }^{2}$, Kang Zou ${ }^{2}$, Chuan Li ${ }^{1,3}$, Tianfu Wen ${ }^{1,3}$ and Xin Sun ${ }^{2 *}$

\begin{abstract}
Background: The controlling nutritional status (CONUT) score has been widely used to evaluate the nutritional and immunological status. Clinical value of postoperative CONUT (PoCONUT) score in hepatocellular carcinoma (HCC) remains unknown. This study assessed whether PoCONUT score could serve as a useful predictor of survival for patients with small HCC.

Methods: 547 consecutive patients with small HCC who underwent liver resection between February 2007 and December 2015 were included in this retrospective case-control study. Patients were categorized into two groups: low PoCONUT group (PoCONUT score $\leq 2, n=382$ ) and high PoCONUT group (PoCONUT score $\geq 3, n=165$ ). Propensity score matching (PSM) analysis was applied to balance the bias in baseline characteristics. A cumulative survival curve was established by the Kaplan-Meier method, and differences in OS and RFS among CONUT score groups were determined by the log rank test. Cox proportional hazard regression analysis was used to evaluate the association of PoCONUT score and overall survival (OS) and recurrence-free survival (RFS), with calculation of hazard ratios (HRs) and $95 \%$ confidence intervals ( $95 \%$ Cls).
\end{abstract}

Results: Cox proportional hazard regression analysis suggested that the PoCONUT score was an independent risk factor for both OS and RFS in patients with small HCC before and after PSM.

Conclusions: High PoCONUT score helps to predict worse OS and RFS in patients with small HCC who underwent liver resection.

Keywords: Controlling nutritional status, Hepatocellular carcinoma, Milan criteria, Liver resection

\section{Background}

$\mathrm{HCC}$ is one of the most common malignancies and remains the third leading cause of cancer-related deaths worldwide [1]. Owing to the high prevalence of hepatitis $B$ virus (HBV), nearly half of the new cases and $\mathrm{HCC}$

\footnotetext{
*Correspondence: sunxin@wchscu.cn

${ }^{2}$ Chinese Evidence-based Medicine Center, West China Hospital, Sichuan University, Chengdu 610041, China

Full list of author information is available at the end of the article
}

related deaths occurred in China [2]. Liver resection is widely accepted as the standard treatment for patients with small HCC meeting Milan Criteria [3-5]. Despite the advances in perioperative management and postoperative prophylactic treatment, the overall prognosis of patients who underwent liver resection remains unsatisfactory [6]. Biological properties of tumor and remnant liver function were the main prognostic factors affecting overall survival and recurrence after liver resection $[7,8]$. 
Recently, nutritional and immunological status was found to be related to the surgical prognosis of many kinds of malignancies $[9,10]$. One of the widely used scoring systems for nutritional and immunological status is the CONUT score, which has the advantage of being readily available from three simple parameters: serum albumin concentration, total lymphocyte count and total cholesterol concentration in peripheral blood [11]. Furthermore, a high preoperative CONUT (PreCONUT) score was reported to be associated not only with postoperative complications but also with the long-term prognosis of patients with HCC who underwent liver resection [12, 13]. However, these studies only focus on the PreCONUT score, the clinical value of PoCONUT score, which may reflect the immunological and nutritional status after surgical removal of the tumor, is largely undefined.

This study was designed to evaluate whether PoCONUT score could serve as a useful prognostic predictor for OS and RFS of patients with small HCC who underwent liver resection.

\section{Method \\ Patients}

Clinical data were retrospectively retrieved for 547 consecutive patients with small HCC meeting Milan criteria who underwent liver resection as an initial treatment between February 2007 and December 2015 in the Department of Liver Surgery \& Liver Transplantation Center of West China Hospital, Sichuan University. Diagnosis of HCC, microvascular invasion (MVI), tumor differentiation was assessed by a postoperative pathological examination. Liver cirrhosis was also determined in postoperative histopathology examination in accordance with the Ishak scoring system [14]. This study was conducted with the approval of the Ethics Committee of West China Hospital, Sichuan University and all methods were performed in accordance with the Declaration of Helsinki.

\section{Definition and cut-off value for CONUT score}

Preoperative blood samples were taken within 1 week before surgery. CONUT score was calculated based on serum albumin concentration, total lymphocyte count and total cholesterol concentration in each patient (Table 1) [11]. In the present study, we defined CONUT score $\leq 2$ as a "low CONUT" group, and CONUT score $\geq 3$ as a "high CONUT" group as previously described $[15,16]$. PoCONUT score was calculated based on results of postoperative blood samples at 1st followup visit, one month after the surgery.
Table 1 Assessment of undernutrition degree by CONUT score

\begin{tabular}{lllll}
\hline Parameters & \multicolumn{5}{l}{ Undernutrition degree } \\
\cline { 2 - 5 } & Normal & Light & Moderate & Severe \\
\hline Serum albumin $(\mathrm{g} / \mathrm{dl})$ & $\geq 3.5$ & $3.0-3.49$ & $2.5-2.99$ & $<2.5$ \\
Score & 0 & 2 & 4 & 6 \\
Total lymphocytes/ml & $>1600$ & $1200-1599$ & $800-1199$ & $<800$ \\
Score & 0 & 1 & 2 & 3 \\
Cholesterol (mg/dl) & $>180$ & $140-180$ & $100-139$ & $<100$ \\
Score & 0 & 1 & 2 & 3 \\
Total score & $0-1$ & $2-4$ & $5-8$ & $9-12$ \\
\hline
\end{tabular}

CONUT controlling nutritional status

\section{Follow-up visit}

Each patient was regularly followed up at the 1st and every 3 months within 3 years after surgery, and every 6 months thereafter. Follow-up examinations included blood cell and differential counts, alpha-fetoprotein (AFP) level, biochemical test combo or liver function test, HBV-DNA level (if the patient was diagnosed with HBV infection), image and physical examinations. When recurrence was suspected, additional examinations such as contrast-enhanced computed tomography (CT) and/ or hepatic angiography were performed. Recurrent HCC were treated with salvage liver transplantation, repeat liver resection, radiofrequency ablation, transcatheter arterial chemoembolization or systemic therapy according to a strategy previous described [17]. RFS time was defined as the time interval between surgery and recurrence, while OS time was defined as the time interval between the surgery and death or last follow-up visit. The last follow-up date was the end of June 2016.

\section{Statistical analysis}

Continuous variables were present as mean \pm standard deviation or median (range) and were compared using independent sample t-test. Categorical data were present as a number (\%) and analyzed using the chi-square test or Fisher's exact test. A cumulative survival curve was established by the Kaplan-Meier method, and differences in OS and RFS among CONUT score groups were determined by the log rank test. Cox proportional hazard regression analysis was used to evaluate the association of CONUT score and OS and RFS, with calculation of HRs and $95 \%$ CIs.

In this retrospective study, a PSM analysis was performed to minimize the selection bias between two groups. The possible clinicopathological variables (age, gender, total bilirubin, prothrombin time, hemoglobin, platelet, white blood cell, total cholesterol, PreCONUT 
score, tumor size, liver cirrhosis, MVI and tumor differentiation) were included in the PSM. Propensity scores were matched using a caliper of 0.1 and a neighbor matching algorithm was applied. A difference $<20 \%$ of absolute value was acceptable $[18,19]$.

All statistical analyses were performed by SPSS software version 26.0 (SPSS company, Chicago, IL, USA). Calculated $\mathrm{P}$ values were two-sided, and a $\mathrm{P}$ value less than 0.05 was considered statistically significant.

\section{Results}

Baseline characters

The clinical and pathologic characters of the patients are summarized in Table 2. Of the 547 patients, $382(69.8 \%)$ had a PoCONUT score $\leq 2$ (low PoCONUT group) and $165(30.2 \%)$ had a PoCONUT score $\geq 3$ (high PoCONUT group). Patients in high PoCONUT group had higher total bilirubin and PreCONUT score, longer prothrombin time, lower hemoglobin, platelet, and white blood cell counts, they also tended to have HBV infection and liver cirrhosis (all $\mathrm{P}<0.05)$. To minimize the difference between the two groups, a PSM analysis was applied. After this matching, 133 matched pairs were created.

Table 3 Comparison of clinical and pathologic characters between two groups according to postoperative CONUT score in propensity score matched pairs

\begin{tabular}{|c|c|c|c|}
\hline Variables & $\begin{array}{l}\text { PoCONUT } \leq 2 \\
N=133\end{array}$ & $\begin{array}{l}\text { PoCONUT } \geq 3 \\
N=133\end{array}$ & $\mathbf{P}$ \\
\hline Age (years) & $51.71 \pm 13.71$ & $48.73 \pm 12.33$ & 0.058 \\
\hline Male/female & $112 / 22$ & $112 / 22$ & 1.000 \\
\hline HBsAg (+/-) & $123 / 10$ & $124 / 9$ & 1.000 \\
\hline $\mathrm{AFP}>400 \mathrm{ng} / \mathrm{ml}$ & $39(29.3 \%)$ & $42(31.6 \%)$ & 0.790 \\
\hline TBIL ( $\mu \mathrm{mol} / \mathrm{L})$ & $15.90 \pm 6.87$ & $15.32 \pm 6.17$ & 0.473 \\
\hline $\mathrm{ALT}(\mathrm{IU} / \mathrm{L})$ & $47.20 \pm 36.02$ & $45.03 \pm 37.64$ & 0.631 \\
\hline AST (IU/L) & $40.86 \pm 23.18$ & $39.77 \pm 29.05$ & 0.735 \\
\hline PT (s) & $12.43 \pm 2.33$ & $12.38 \pm 1.22$ & 0.823 \\
\hline CREA ( $\mu \mathrm{mol} / \mathrm{L})$ & $76.03 \pm 14.85$ & $75.07 \pm 16.50$ & 0.616 \\
\hline $\mathrm{HGB}(\mathrm{g} / \mathrm{L})$ & $142.37 \pm 17.45$ & $142.61 \pm 17.35$ & 0.910 \\
\hline $\mathrm{PLT}(* 10 \wedge 9 / \mathrm{L})$ & $101.99 \pm 41.30$ & $103.24 \pm 38.99$ & 0.800 \\
\hline WBC $\left(*^{*} 10 \wedge 9 / L\right)$ & $4.86 \pm 1.74$ & $4.80 \pm 1.61$ & 0.773 \\
\hline Albumin (g/dL) & $41.55 \pm 6.10$ & $41.98 \pm 4.39$ & 0.461 \\
\hline Total cholesterol (mg/dl) & $147.90 \pm 28.77$ & $145.90 \pm 27.63$ & 0.745 \\
\hline PreCONUT $\leq 2$ & $51(38.3 \%)$ & $50(37.6 \%)$ & 1.000 \\
\hline Tumor size $<3$ cm & $72(54.1 \%)$ & $63(47.4 \%)$ & 0.327 \\
\hline Solitary tumor & $113(85.0 \%)$ & $117(88.0 \%)$ & 0.591 \\
\hline Blood loss (mL) & $355.64 \pm 317.88$ & $358.12 \pm 273.78$ & 0.946 \\
\hline Transfusion (+/-) & $9 / 124$ & $9 / 124$ & 0.159 \\
\hline $\begin{array}{l}\text { Operation type } \\
\text { major/minor }\end{array}$ & $21 / 122$ & $22 / 111$ & 0.986 \\
\hline Operation duration (min) & $226.46 \pm 60.89$ & $219.22 \pm 65.56$ & 0.389 \\
\hline Cirrhosis (+/-) & $119 / 14$ & $119 / 14$ & 1.000 \\
\hline $\mathrm{MVI}(+/-)$ & $27 / 106$ & $27 / 106$ & 1.000 \\
\hline Poor differentiation & $58(43.6 \%)$ & $48(36.2 \%)$ & 0.260 \\
\hline Hospital stay $(\mathrm{d})$ & $11.05 \pm 5.07$ & $11.67 \pm 4.78$ & 0.875 \\
\hline
\end{tabular}

Data are displayed as mean \pm standard deviation, or number of patients (percentage)

CONUT controlling nutritional status, PreCONUT preoperative CONUT, PoCONUT postoperative CONUT, HBsAg hepatitis $B$ viral surface antigen, AFP alpha-fetoprotein, TBIL total bilirubin, ALT alanine aminotransferase, AST aspartate aminotransferase, $P T$ prothrombin time, CREA creatinine, HGB hemoglobin, PLT platelet count, WBC white blood cell count, $M V I$ microvascular invasion 
The baseline features were comparable between the two groups in the matched cohort (Table 3).

\section{Impact of PreCONUT score on OS and RFS}

After a median follow-up time of 34 months, 223 (40.8\%) patients were found recurrent and 128 (23.4\%) patients died. 1-, 3-, 5-year estimated OS rates of patients in low PreCONUT group were $94.8 \%, 80.2$ and $68.8 \%$ respectively, and $95.4 \%, 74.9$ and $59.2 \%$ respectively for patients in high PreCONUT group (log-rank test, $\mathrm{P}=0.134$, Fig. 1A). 1-, 3-, 5-year estimated RFS rates of patients in low PreCONUT group were $78.8 \%, 62.5$ and $46.5 \%$ respectively, and $77.4 \%, 50.3$ and $46.2 \%$ respectively for patients in high PreCONUT group (log-rank test, $\mathrm{P}=0.296$, Fig. 1B).

\section{Impact of PoCONUT score on OS and RFS}

Before PSM, the 1-, 3-, 5-year estimated OS rates of patients in low PoCONUT group were 96.7\%, 83.6 and $72.8 \%$ respectively, and $91.3 \%, 64.7$ and $48.2 \%$ respectively for patients in high PoCONUT group (log-rank test, $\mathrm{P}<0.001$, Fig. 2 A). 1-, 3-, 5-year estimated RFS rates of patients in low PoCONUT group were $81.2 \%, 63.3$ and $51.4 \%$ respectively, and $68.3 \%, 43.4$ and $33.4 \%$ respectively for patients in high PoCONUT group (log-rank test, $\mathrm{P}<0.001$, Fig. 2B).

After PSM, 1-, 3-, 5-year estimated OS rates of patients in low PoCONUT group were 95.4\%, 81.2 and $63.3 \%$ respectively, and $88.7 \%, 63.0$ and $44.2 \%$ respectively for patients in high PoCONUT group (log-rank test, $\mathrm{P}=0.009$, Fig. 3A). 1-, 3-, 5-year estimated RFS rates of patients in low PoCONUT group were $80.4 \%, 57.5$ and
$49.5 \%$ respectively, and $66.1 \%, 40.3$ and $31.0 \%$ respectively for patients in high PoCONUT group (log-rank test, $\mathrm{P}=0.015$, Fig. 3B).

\section{Risk factors of prognosis}

Univariate and multivariate analyses were carried out to identify the risk factors of prognosis. As for OS, thirteen potential covariates with $\mathrm{p}$ values less than 0.10 in univariate analysis were further included in Cox regression analysis. And Cox regression analysis suggested that elevated AFP $(\mathrm{P}=0.038$, hazard ratio $(\mathrm{HR})=1.622,95 \%$ confidence interval $(\mathrm{CI}) 1.028-2.560)$, MVI $(\mathrm{P}=0.007$, $\mathrm{HR}=1.929,95 \% \mathrm{CI} 1.202-3.098)$ and PoCONUT score $(\mathrm{P}=0.019, \mathrm{HR}=1.708,95 \% \mathrm{CI} 1.091-2.676)$ were independent risk factors for reduced OS (Table 4).

As for RFS, ten potential covariates with $\mathrm{p}$ values less than 0.10 in univariate analysis were further included in Cox regression analysis.Poor differentiation $(\mathrm{P}=0.014$, $\mathrm{HR}=1.588$, 95 \%CI 1.098-2.298), tumor number $(\mathrm{P}=0.049, \quad \mathrm{HR}=1.611, \quad 95 \quad \% \mathrm{CI} 1.003-2.589), \quad \mathrm{MVI}$ $(\mathrm{P}=0.001, \mathrm{HR}=1.963,95 \% \mathrm{CI} 1.314-2.933)$ and $\mathrm{PoCO}-$ NUT score $(\mathrm{P}=0.008, \mathrm{HR}=1.639,95 \% \mathrm{CI} 1.139-2.358)$ were prognostic factors for reduced RFS in multivariate analysis (Table 5).

\section{Discussion}

In the present study, multivariate analysis found that the high PoCONUT score $(\geq 3)$ was an independent prognostic factor for both OS and RFS in patients with small HCC who underwent liver resection. Researchers have established the prognostic value of the PreCONUT score in multiple malignant tumors [12, 20,21]. To our
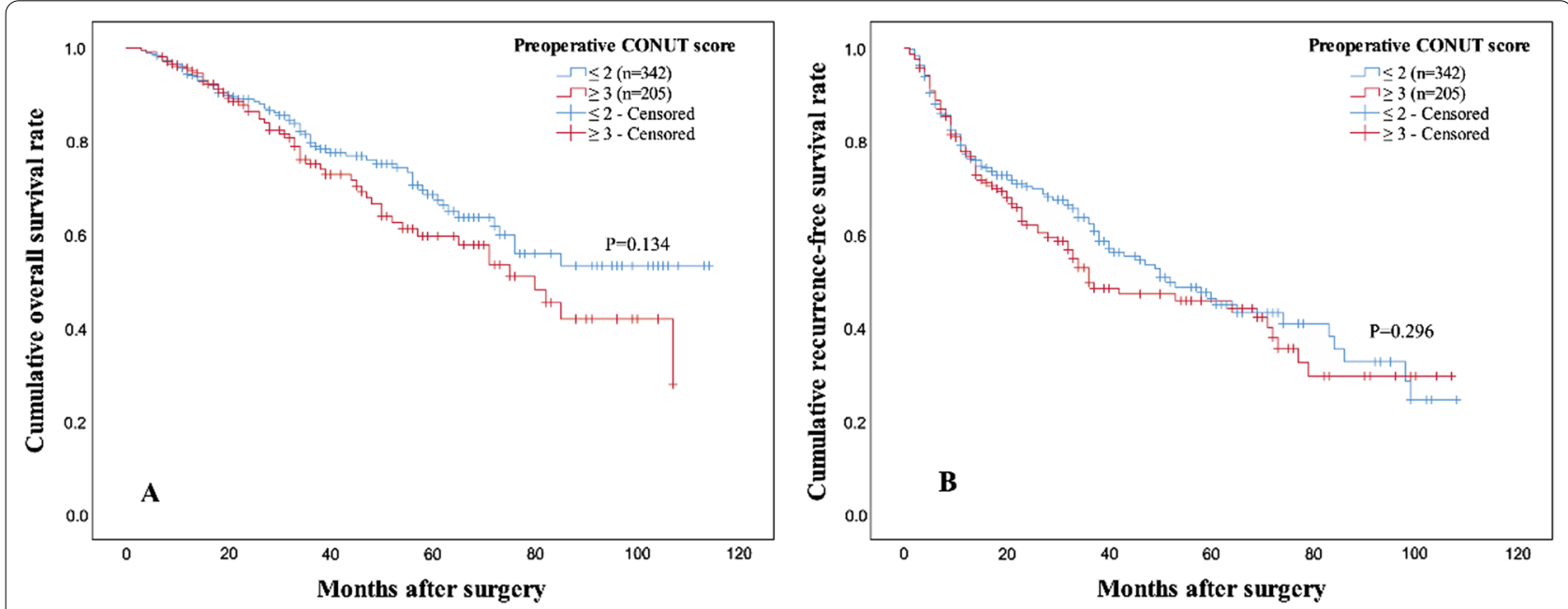

Fig. 1 Kaplan-Meier curves of OS (A) and RFS (B) for patients in high and low preoperative CONUT score groups. There was no difference in cumulative OS or RFS rates of patients with a high and low preoperative CONUT score. OS overall survival, RFS recurrence-free survival, CONUT controlling nutritional status 

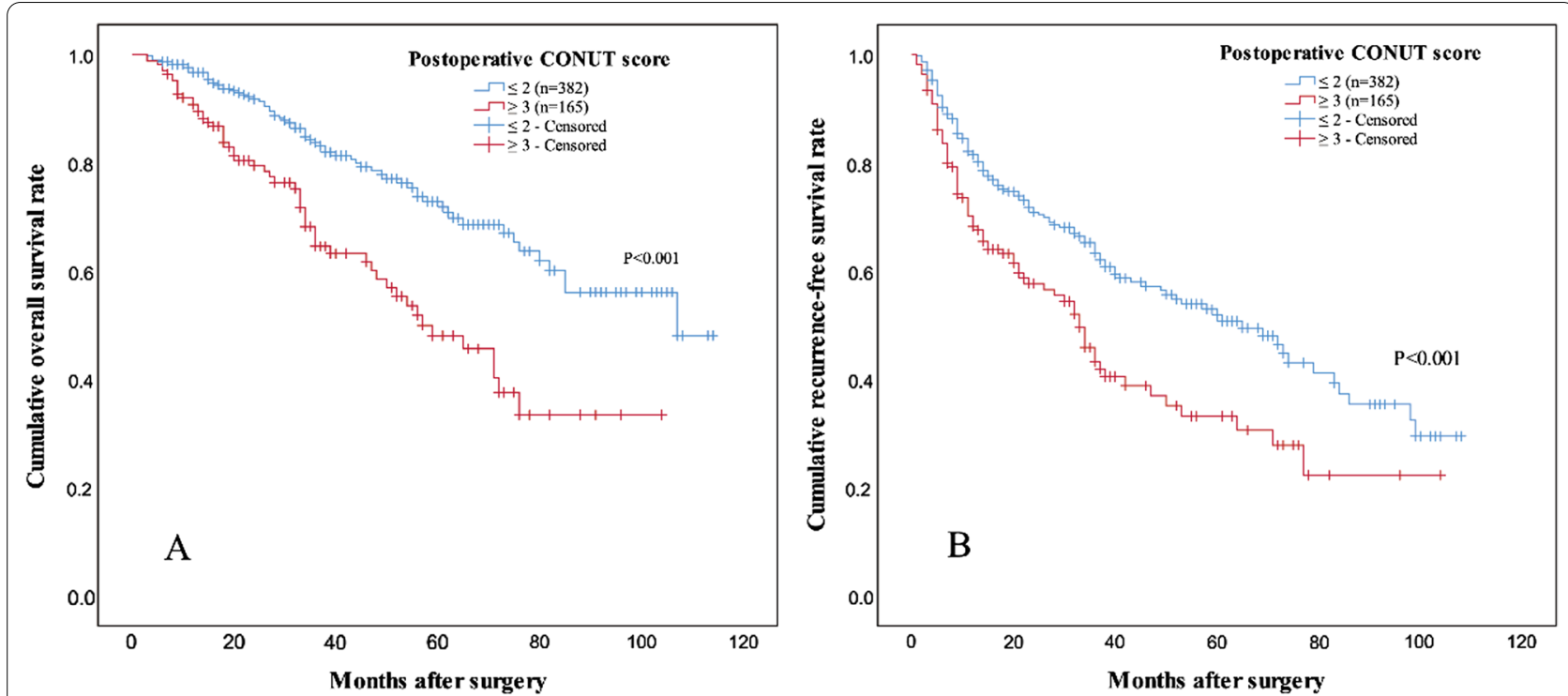

Fig. 2 Kaplan-Meier curves of OS (A) and RFS (B) for patients with a high or low postoperative CONUT score before propensity score matching. Patients with a high postoperative CONUT score had a decreased OS and RFS rate. OS overall survival, RFS recurrence-free survival, CONUT controlling nutritional status
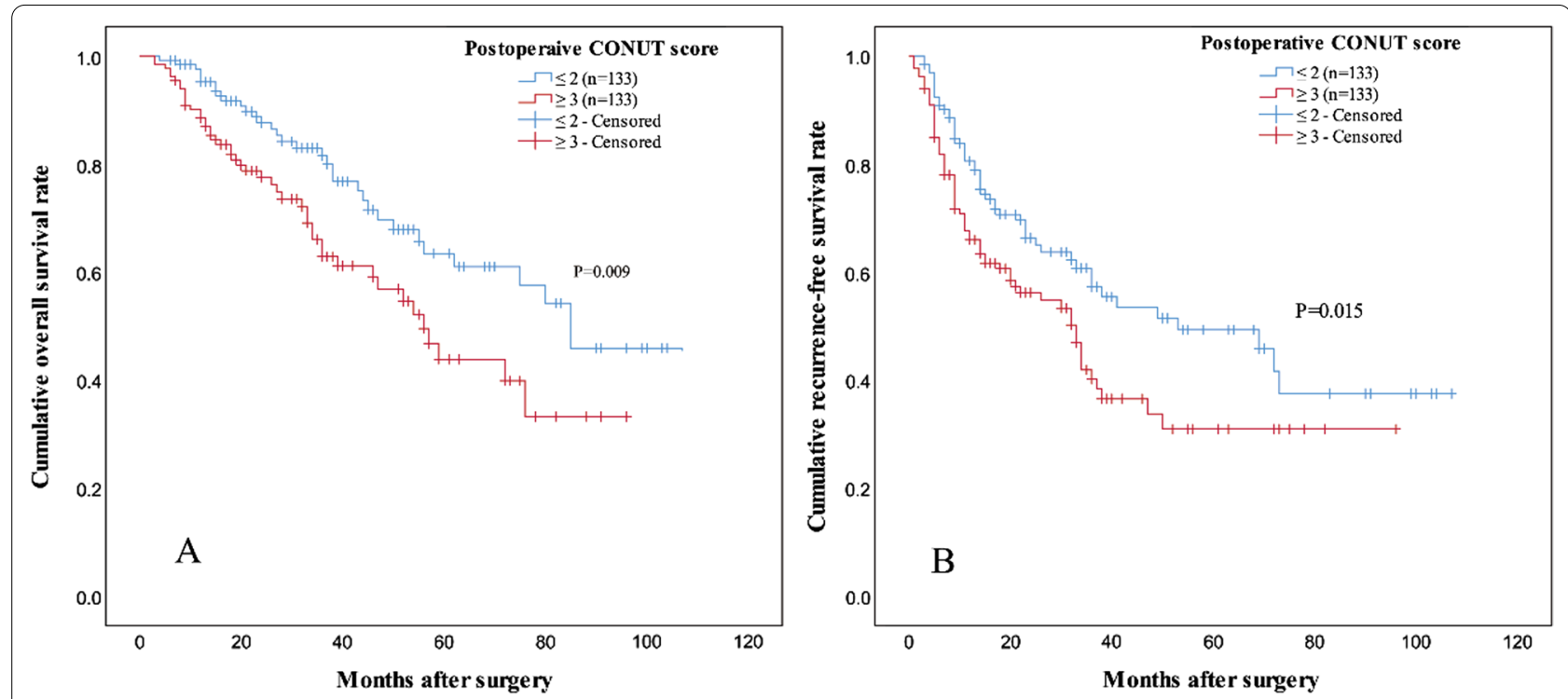

Fig. 3 Kaplan-Meier curves of OS (A) and RFS (B) for patients with a high or low postoperative CONUT score after propensity score matching. Patients with a high postoperative CONUT score had a decreased OS and RFS rate. OS overall survival, RFS recurrence-free survival, CONUT controlling nutritional status

knowledge, this is the first study to investigate the relationship between the PoCONUT score and the prognosis of patients with small $\mathrm{HCC}$ who underwent liver resection using a PSM analysis.

CONUT score was originally used in early detection and continuous control for hospital undernutrition [11]. It is derived from three readily available parameters, serum albumin concentration, total lymphocyte count and total cholesterol concentration. Among them, serum albumin is not only a major indicator of nutritional status but also affected by chronic inflammation, liver function reserve and body fluid change [22, 23]. Hypoalbuminemia was reported to be correlated with immunosuppression and poor prognosis of malignancies [24]. Total 
Table 4 Univariate and multivariate analyses of prognostic factors for overall survival in a propensity score matched cohort

\begin{tabular}{|c|c|c|c|c|}
\hline \multirow[t]{2}{*}{ Factors } & \multicolumn{2}{|l|}{ Univariate analysis } & \multicolumn{2}{|c|}{ Multivariate analysis } \\
\hline & $\mathrm{HR}(95 \% \mathrm{Cl})$ & $\mathbf{P}$ & $\mathrm{HR}(95 \% \mathrm{Cl})$ & $\mathbf{P}$ \\
\hline Age & 0.989 (0.969-1.009) & 0.269 & & \\
\hline Gender (male) & $1.313(0.695-2.481)$ & 0.402 & & \\
\hline $\operatorname{HBsAg}(+)$ & $1.627(0.512-5.168)$ & 0.409 & & \\
\hline $\operatorname{AFP}(>400 \mathrm{ng} / \mathrm{mL})$ & $1.694(1.073-2.675)$ & 0.024 & $1.622(1.028-2.560)$ & 0.038 \\
\hline TBIL & $0.999(0.960-1.040)$ & 0.959 & & \\
\hline ALT & $1.010(1.003-1.018)$ & 0.007 & & \\
\hline AST & $1.010(1.000-1.020)$ & 0.052 & & \\
\hline PT & $1.069(0.930-1.229)$ & 0.346 & & \\
\hline CREA & 1.017 (0.999-1.035) & 0.061 & & \\
\hline HGB & $1.003(0.988-1.019)$ & 0.663 & & \\
\hline PLT & $0.997(0.991-1.004)$ & 0.425 & & \\
\hline WBC & $0.942(0.799-1.111)$ & 0.476 & & \\
\hline $\operatorname{PreCONUT}(\geq 3)$ & $0.775(0.499-1.204)$ & 0.257 & & \\
\hline Tumor size $(3-5 \mathrm{~cm})$ & $0.970(0.626-1.501)$ & 0.890 & & \\
\hline Tumor number (2-3) & 1.589 (0.919-2.748) & 0.097 & & \\
\hline Blood loss & $1.001(1.000-1.002)$ & 0.033 & & \\
\hline Transfusion (+) & $1.854(0.921-3.735)$ & 0.084 & & \\
\hline Cirrhosis (+) & $1.502(0.607-3.720)$ & 0.379 & & \\
\hline Operation duration & $1.078(0.916-1.268)$ & 0.367 & & \\
\hline Operation type (major) & $1.139(0.797-1.626)$ & 0.473 & & \\
\hline $\mathrm{MVI}(+)$ & $2.210(1.390-3.514)$ & 0.001 & $1.929(1.202-3.098)$ & 0.007 \\
\hline Poor differentiation (+) & $1.287(0.810-2.044)$ & 0.285 & & \\
\hline Hospital stay & $1.060(0.771-1.456)$ & 0.720 & & \\
\hline PoCONUT $(\geq 3)$ & $1.805(1.155-2.820)$ & 0.009 & $1.708(1.091-2.676)$ & 0.019 \\
\hline
\end{tabular}

CONUT controlling nutritional status, PreCONUT preoperative CONUT, PoCONUT postoperative CONUT, HBsAg hepatitis B viral surface antigen, AFP alpha-fetoprotein, TBIL total bilirubin, ALT alanine aminotransferase, AST aspartate minotransferase, PT prothrombin time, CREA creatinine, HGB hemoglobin, PLT platelet count, WBC white blood cell count, MVImicrovascular invasion, HR hazard ratio, CI confidence interval

cholesterol concentration is another indicator of nutritional status. A decreased concentration of cholesterol indicates not only a calorie deficiency but also that the cell is being deprived of an essential nutrient required to maintain metabolic equilibrium and membrane integrity [25]. And total cholesterol was further reported to be an independent risk factor of poor OS and RFS for patients with HCC [26]. Lymphocyte, another component of the CONUT score, plays a significant role in anti-tumor immunity [27]. Besides, lymphocyte and lymphocyterelated indexes were found to be related to the survival of patients with HCC $[28,29]$. Therefore, CONUT score theoretically reflects the immunological and nutritional status of patients with HCC.

Takagi $\mathrm{K}$ et al. reported that a PreCONUT score $\geq 3$ was a reliable and independent predictor of poor survival after liver resection for patients with HCC in 2017 [16]. Similarly, some other literature found a high PreCONUT score could help to predict the survival of patients with $\mathrm{HCC}[15,30]$. In the present study, the PreCONUT score had a trend to be related with the survival of HCC patients, but the difference was not statistically significant. We believe this might be caused by the bias of patient selection. In the previous three studies, they enrolled 43 (20.6\%) patients of BCLC tumor stage C, 133 (45.1\%) patients of TNM stage III/IV and $112(31.4 \%)$ patients of TNM stage III/IV respectively $[15,16,30]$. However, all the patients enrolled in the present study were classified in BCLC tumor stage 0/A. We hypothesized that patients of early tumor stage represented a better immunological and nutritional status when compared to those in mediate/advanced stage. The PreCONUT score may not able to serve as a stable predictor for the survival of patients with small HCC meeting Milan criteria.

In the present study, for the first time, we investigated the clinical value of PoCONUT score, which could reflect the actual immunological and nutritional status after surgical removal of the tumor, and we found PoCONUT score was a stable predictor for both OS and RFS in patients with small HCC after liver resection. In Chinese tradition, a patient would have a very good rest and 
Table 5 Univariate and multivariate analyses of prognostic factors for recurrence-free survival in a propensity score matched cohort

\begin{tabular}{|c|c|c|c|c|}
\hline \multirow[t]{2}{*}{ Factors } & \multicolumn{2}{|l|}{ Univariate analysis } & \multicolumn{2}{|c|}{ Multivariate analysis } \\
\hline & $\mathrm{HR}(95 \% \mathrm{Cl})$ & $\mathbf{P}$ & $\mathrm{HR}(95 \% \mathrm{Cl})$ & $\mathbf{P}$ \\
\hline Age & $0.996(0.977-1.014)$ & 0.638 & & \\
\hline Gender (male) & $1.449(0.855-2.455)$ & 0.168 & & \\
\hline $\operatorname{HBsAg}(+)$ & $1.439(0.633-3.272)$ & 0.385 & & \\
\hline $\operatorname{AFP}(>400 \mathrm{ng} / \mathrm{mL})$ & $1.310(0.896-1.915)$ & 0.163 & & \\
\hline TBIL & $0.998(0.962-1.036)$ & 0.919 & & \\
\hline ALT & $1.008(1.001-1.016)$ & 0.030 & & \\
\hline AST & $1.013(1.002-1.024)$ & 0.025 & & \\
\hline PT & $1.093(0.933-1.280)$ & 0.271 & & \\
\hline CREA & $1.017(1.001-1.034)$ & 0.039 & & \\
\hline HGB & $1.002(0.988-1.016)$ & 0.832 & & \\
\hline PLT & $0.997(0.991-1.003)$ & 0.387 & & \\
\hline WBC & $0.951(0.821-1.102)$ & 0.951 & & \\
\hline PreCONUT $(\geq 3)$ & $0.911(0.631-1.317)$ & 0.622 & & \\
\hline Tumor size $(3-5 \mathrm{~cm})$ & $0.939(0.656-1.344)$ & 0.731 & & \\
\hline Tumor number (2-3) & $1.684(1.050-2.701)$ & 0.030 & $1.611(1.003-2.589)$ & 0.049 \\
\hline Blood loss & $1.001(0.998-1.002)$ & 0.834 & & \\
\hline Transfusion (+) & $1.167(0.898-3.110)$ & 0.105 & & \\
\hline Cirrhosis (+) & $1.352(0.707-2.585)$ & 0.363 & & \\
\hline Operation duration & $1.072(0.864-1.582)$ & 0.461 & & \\
\hline Operation type (major) & $1.212(0.889-1.634)$ & 0.573 & & \\
\hline $\mathrm{MVI}(+)$ & $2.169(1.461-3.221)$ & $<0.001$ & $1.963(1.314-2.933)$ & 0.001 \\
\hline Poor differentiation (+) & $1.579(1.095-2.277)$ & 0.014 & $1.588(1.098-2.298)$ & 0.014 \\
\hline Hospital stay & $1.004(0.984-1.021)$ & 0.641 & & \\
\hline PoCONUT $(\geq 3)$ & $1.561(1.088-2.239)$ & 0.015 & $1.639(1.139-2.358)$ & 0.008 \\
\hline
\end{tabular}

nutritional support immediately after any kinds of surgery. If a high PoCONUT score emerges with such adequate support, it indicates that the patient's poor ability and potential to recover from surgery which promotes tumoral recurrence. In the present study, we used a PSM analysis to balance the baseline covariates between high and low PoCONUT groups. It is used in many articles to balance treated groups across all risk factors [12, 19] Interestingly, before PSM, a high PoCONUT score was significantly associated with several host-related factors including higher total bilirubin and PreCONUT score, longer prothrombin time, lower hemoglobin, platelet, and white blood cell counts and higher incidence of liver cirrhosis. These results validated our hypothesis that patients with a high PoCONUT score had a worse potential and ability to recover from surgery.

There is no universally accepted cut-off value of CONUT score for now. High CONUT score was defined as CONUT score $\geq 3$ in the present study which was consistent with some previous reports [15, 16]. However, Harimoto $\mathrm{N}$ et al. defined the high CONUT score as CONUT score $\geq 4$ using a receiver operating characteristic (ROC) analysis [12]. We noticed that the mean age of the study population was lower (49.8 VS 68.6) in the present study. And age is positively related to CONUT score [31]. Only $79(14.4 \%)$ patients had a PoCONUT score $\geq 4$ in the present study. Besides, we tried to identify more patients who were at risk of immunosuppression and undernutrition with a cut-off value of 3 . Thirdly, the cut-off value for the PoCONUT score associated with survival using ROC analysis in the present study was also 3 (area under the curve $=0.627$, data not shown). Further study is needed to determine the adequate cut-off value of the CONUT score to predict prognosis.

The present study has several limitations. First, this was a single-center, retrospective study, there might be potential selection bias. Second, the predominant etiology in the present study is $\mathrm{HBV}$ while most $\mathrm{HCC}$ cases are related to hepatitis $C$ Virus and alcohol in western countries and Japan. So, the results of the present study should be further validated by a different etiological population. 


\section{Conclusions}

High PoCONUT score helps to predict worse OS and RFS in patients with small HCC who underwent liver resection.

\begin{abstract}
Abbreviations
HCC: Hepatocellular carcinoma; OS: Overall survival; RFS: Recurrence free survival; HR: Hazard ratio; Cl: Confidential interval; HBV: Hepatitis B virus; MVI: Microvascular invasion; AFP: Alpha fetal protein; CT: Computed tomography.
\end{abstract}

\section{Acknowledgements}

Not applicable.

\section{Authors' contributions}

WP and XS offered the idea of this study, KZ collected and analyzed the patient data. WP drafted the manuscript. WP and MHY performed the statistical analysis. TFW was the supervisor of this study and was a major contributor in revising the manuscript. All authors read and approved the final manuscript.

\section{Funding}

This article was supported by National Natural Science Foundation of China (Grant No. 81590955). The funders had no role in study design, data collection and analysis, decision to publish, or preparation of the manuscript.

\section{Availability of data and materials}

The datasets generated and analyzed during the current study are not publicly available due to patient privacy but are available from the corresponding author on reasonable request.

\section{Declarations}

\section{Ethics approval and consent to participate}

The Ethics Committee of West China Hospital, Sichuan University approved this study design and waived the need of informed consent due to the retrospective nature of the study.

\section{Consent for publication}

Not applicable.

\section{Competing interests}

The authors declare that they have no competing interests.

\section{Author details}

${ }^{1}$ Department of Liver Surgery, West China Hospital, Sichuan University, Chengdu 610041, China. ${ }^{2}$ Chinese Evidence-based Medicine Center, West China Hospital, Sichuan University, Chengdu 610041, China. ${ }^{3}$ Laboratory of Liver Transplantation, West China Hospital, Sichuan University, Chengdu 610041, China.

Received: 26 February 2021 Accepted: 28 August 2021

Published online: 07 September 2021

\section{References}

1. Bray F, Ferlay J, Soerjomataram I, Siegel RL, Torre LA, Jemal A. Global cancer statistics 2018: GLOBOCAN estimates of incidence and mortality worldwide for 36 cancers in 185 countries. CA Cancer J Clin. 2018;68(6):394-424.

2. Feng RM, Zong YN, Cao SM, Xu RH. Current cancer situation in China: good or bad news from the 2018 Global Cancer Statistics? Cancer Commun (Lond). 2019:39(1):22.

3. Kokudo N, Hasegawa K, Akahane M, Igaki H, Izumi N, Ichida T, Uemoto S, Kaneko S, Kawasaki S, Ku Y, et al. Evidence-based Clinical Practice Guidelines for Hepatocellular Carcinoma: The Japan Society of Hepatology 2013 update (3rd JSH-HCC Guidelines). Hepatol Res. 2015;45:2.
4. Zhou J, Sun HC, Wang Z, Cong WM, Wang JH, Zeng MS, Yang JM, Bie P, Liu LX, Wen TF, et al. Guidelines for diagnosis and treatment of primary liver cancer in China (2017) edition. Liver Cancer. 2018;7(3):235-60.

5. Forner A, Llovet JM, Bruix J. Hepatocellular carcinoma. Lancet (London, England). 2012;379(9822):1245-55.

6. Poon RT, Fan ST, Lo CM, Liu CL, Wong J. Long-term survival and pattern of recurrence after resection of small hepatocellular carcinoma in patients with preserved liver function: implications for a strategy of salvage transplantation. Ann Surg. 2002;235(3):373-82.

7. Ercolani G, Grazi GL, Ravaioli M, Del Gaudio M, Gardini A, Cescon M, Varotti G, Cetta F, Cavallari A. Liver resection for hepatocellular carcinoma on cirrhosis: univariate and multivariate analysis of risk factors for intrahepatic recurrence. Ann Surg. 2003;237(4):536-43.

8. Ikai I, Arii S, Kojiro M, Ichida T, Makuuchi M, Matsuyama Y, Nakanuma Y, Okita K, Omata M, Takayasu K, et al. Reevaluation of prognostic factors for survival after liver resection in patients with hepatocellular carcinoma in a Japanese nationwide survey. Cancer. 2004;101(4):796-802.

9. Schwegler I, von Holzen A, Gutzwiller JP, Schlumpf R, Muhlebach S, Stanga Z. Nutritional risk is a clinical predictor of postoperative mortality and morbidity in surgery for colorectal cancer. Br J Surg. 2010;97(1):92-7.

10. Ray-Coquard I, Cropet C, Van Glabbeke M, Sebban C, Le Cesne A, Judson I, Tredan O, Verweij J, Biron P, Labidi I, et al. Lymphopenia as a prognostic factor for overall survival in advanced carcinomas, sarcomas, and lymphomas. Can Res. 2009;69(13):5383-91.

11. de Ulibarri J, Gonzalez-Madrono A, de Villar NG, Gonzalez P, Gonzalez B, Mancha A, Rodriguez F, Fernandez G. CONUT: a tool for controlling nutritional status. First validation in a hospital population. Nutr Hosp. 2005;20(1):38-45.

12. Harimoto N, Yoshizumi T, Inokuchi S, Itoh S, Adachi E, Ikeda Y, Uchiyama H, Utsunomiya T, Kajiyama K, Kimura K, et al. Prognostic significance of preoperative controlling nutritional status (CONUT) score in patients undergoing hepatic resection for hepatocellular carcinoma: a multiinstitutional study. Ann Surg Oncol. 2018;25(11):3316-23.

13. Takagi K, Umeda Y, Yoshida R, Nobuoka D, Kuise T, Fushimi T, Fujiwara T, Yagi T. Preoperative controlling nutritional status score predicts mortality after hepatectomy for hepatocellular carcinoma. Dig Surg. 2019;36(3):226-32.

14. Ishak K, Baptista A, Bianchi L, Callea F, De Groote J, Gudat F, Denk H, Desmet V, Korb G, MacSween RN, et al. Histological grading and staging of chronic hepatitis. J Hepatol. 1995;22(6):696-9.

15. Wang XB, Chen J, Xiang BD, Wu FX, Li LQ. High CONUT score predicts poor survival and postoperative HBV reactivation in HBV-related hepatocellular carcinoma patients with low HBV-DNA levels. Eur J Surg Oncol. 2019;45(5):782-7.

16. Takagi K, Yagi T, Umeda Y, Shinoura S, Yoshida R, Nobuoka D, Kuise T, Araki $\mathrm{H}$, Fujiwara T. Preoperative Controlling Nutritional Status (CONUT) score for assessment of prognosis following hepatectomy for hepatocellular carcinoma. World J Surg. 2017;41(9):2353-60.

17. Wen T, Jin C, Facciorusso A, Donadon M, Han HS, Mao Y, Dai C, Cheng S, Zhang B, Peng B, et al. Multidisciplinary management of recurrent and metastatic hepatocellular carcinoma after resection: an international expert consensus. Hepatobiliary surgery and nutrition. 2018;7(5):353-71.

18. D'Agostino RB Jr. Propensity score methods for bias reduction in the comparison of a treatment to a non-randomized control group. Stat Med. 1998;17(19):2265-81.

19. Shen J, Liu J, Li C, Wen T, Yan L, Yang J. The impact of tumor differentiation on the prognosis of HBV-associated solitary hepatocellular carcinoma following hepatectomy: a propensity score matching analysis. Dig Dis Sci. 2018;63(7):1962-9.

20. Ryo S, Kanda M, Ito S, Mochizuki Y, Teramoto H, Ishigure K, Murai T, Asada T, Ishiyama A, Matsushita $\mathrm{H}$, et al. The controlling nutritional status score serves as a predictor of short- and long-term outcomes for patients with stage 2 or 3 gastric cancer: analysis of a multi-institutional data set. Ann Surg Oncol. 2019:26(2):456-64.

21. Iseki Y, Shibutani M, Maeda K, Nagahara H, Ohtani H, Sugano K, Ikeya T, Muguruma K, Tanaka H, Toyokawa T, et al. Impact of the Preoperative Controlling Nutritional Status (CONUT) Score on the Survival after Curative Surgery for Colorectal Cancer. PLoS ONE. 2015;10(7):e0132488.

22. Cho HJ, Kim B, Lee JD, Kang DR, Kim JK, Lee JH, Shin SJ, Lee KM, Yoo BM, Lee KJ, et al. Development of risk prediction model for hepatocellular 
carcinoma progression of indeterminate nodules in hepatitis $B$ virusrelated cirrhotic liver. Am J Gastroenterol. 2017;112(3):460-70.

23. Tanriverdi $\mathrm{O}$. A discussion of serum albumin level in advanced-stage hepatocellular carcinoma: a medical oncologist's perspective. Med Oncol. 2014;31(11):282.

24. Roxburgh CS, McMillan DC. Role of systemic inflammatory response in predicting survival in patients with primary operable cancer. Future Oncol. 2010;6(1):149-63

25. Schatz IJ, Masaki K, Yano K, Chen R, Rodriguez BL, Curb JD. Cholesterol and all-cause mortality in elderly people from the Honolulu Heart Program: a cohort study. Lancet (London, England). 2001;358(9279):351-5.

26. Jiang SS, Weng DS, Jiang L, Zhang YJ, Pan K, Pan QZ, Chen CL, Zhao JJ, Zhang XF, Zhang HX, et al. The clinical significance of preoperative serum cholesterol and high-density lipoprotein-cholesterol levels in hepatocellular carcinoma. J Cancer. 2016;7(6):626-32.

27. Chew V, Tow C, Teo M, Wong HL, Chan J, Gehring A, Loh M, Bolze A, Quek $\mathrm{R}$, Lee VK, et al. Inflammatory tumour microenvironment is associated with superior survival in hepatocellular carcinoma patients. J Hepatol. 2010;52(3):370-9.

28. Mano Y, Shirabe K, Yamashita Y, Harimoto N, Tsujita E, Takeishi K, Aishima S, Ikegami T, Yoshizumi T, Yamanaka T, et al. Preoperative neutrophil-to-lymphocyte ratio is a predictor of survival after hepatectomy for hepatocellular carcinoma: a retrospective analysis. Ann Surg. 2013;258(2):301-5.

29. Nagai S, Abouljoud MS, Kazimi M, Brown KA, Moonka D, Yoshida A. Peritransplant lymphopenia is a novel prognostic factor in recurrence of hepatocellular carcinoma after liver transplantation. Transplantation. 2014;97(6):694-701.

30. Harimoto N, Yoshizumi T, Sakata K, Nagatsu A, Motomura T, Itoh S, Harada $\mathrm{N}$, Ikegami T, Uchiyama H, Soejima Y, et al. Prognostic significance of preoperative controlling nutritional status (CONUT) score in patients undergoing hepatic resection for hepatocellular carcinoma. World J Surg. 2017:41(11):2805-12

31. de Ulibarri Perez JI, Fernandez G, Rodriguez Salvanes F, Diaz Lopez AM. Nutritional screening; control of clinical undernutrition with analytical parameters. Nutr Hosp. 2014;29(4):797-811.

\section{Publisher's Note}

Springer Nature remains neutral with regard to jurisdictional claims in published maps and institutional affiliations.
Ready to submit your research? Choose BMC and benefit from:

- fast, convenient online submission

- thorough peer review by experienced researchers in your field

- rapid publication on acceptance

- support for research data, including large and complex data types

- gold Open Access which fosters wider collaboration and increased citations

- maximum visibility for your research: over $100 \mathrm{M}$ website views per year

At BMC, research is always in progress.

Learn more biomedcentral.com/submissions 